\title{
Surface Acoustic Wave Technology and Its Application
}

\section{Gaohui Li}

School of Automotive Engineering, Shanghai University of Engineering Science, Shanghai, China

Email: ligaohui011@126.com

How to cite this paper: Li, G.H. (2017) Surface Acoustic Wave Technology and Its Application. Open Access Library Journal, 4: e4106.

https://doi.org/10.4236/oalib.1104106

Received: November 1, 2017

Accepted: November 27, 2017

Published: November 30, 2017

Copyright $\odot 2017$ by author and Open Access Library Inc.

This work is licensed under the Creative Commons Attribution International License (CC BY 4.0).

http://creativecommons.org/licenses/by/4.0/

\section{(c) (i) Open Access}

\begin{abstract}
This paper introduces briefly the structures, specifications and applications of surface acoustic wave (SAW) sensors, as well as the progresses in the related studies. The SAW sensors include Rayleigh wave, shear horizontal acoustic plate wave, Love wave and Lamb wave sensors. The application fields are classified according to the functions into physical sensors and chemical/biological sensors; the former can be performed more easily, but the latter homes special functions and so needs to be studied in more details based on the practical conditions. The SAW sensors have very high sensitivity and wide applications due to the velocities and phase of SAW are sensitive to the parameter changes of environments.
\end{abstract}

\section{Subject Areas}

Chemical Engineering \& Technology

\section{Keywords}

Surface Acoustic Wave, Sensor, Piezoelectric Substrate

\section{Introduction}

A surface acoustic wave (SAW), referred to as SAW, and is an elastic wave propagating along an elastic solid surface or interface. SAW technology is a set of acoustic, light learning, electronics and micro-processing technology combined with a new cross-disciplinary. Surface acoustic wave is the elastic wave generated and propagated on the surface of the piezoelectric substrate material, and its amplitude is as the depth of the piezoelectric substrate material increases rapidly and decreases. In 1885, the British physicist, Lord Rayleigh, accords to the study of seismic waves. To commemorate him, posterity named the sound waves as lamb waves. In addition to the wave and the shear wave belonging to 
the acoustic wave, there is another form of wave, which is surface acoustic wave, also known as Rayleigh wave. But for a long time it did not cause the scientists interest, which it has no practical application in industry. Until 1965, White and Waltermer et al. pointed out by sedimentation interdigital transducers on piezoelectric substrates interdigital transducer, referred to as IDT, can be effectively motivated and seized surface acoustic wave. And the proper design of the interdigital transducer can make the SAW device has a very good performance. At this point, SAW devices are officially onto the electronics stage. Later, Europe and the United States have invested a lot of manpower, material and financial research in development of surface acoustic wave devices for military and communications, such as delay lines in radar, pulse compression filtering and high temperature resonators.

In addition to the surface acoustic wave along the surface of the piezoelectric substrate, but also has many advantages [1]:

1) SAW propagation on a piezoelectric substrate has a very low propagation velocity and a very short wavelength. And their respective transmission over the corresponding electromagnetic waves speed and wavelength is five orders of magnitude. The use of this feature not only can greatly reduce the size of electronic devices and weight reduction light, to achieve the miniaturization of electronic devices, but also make its performance greatly improved.

2) As the surface acoustic wave is along the solid surface of the body is propagated, and the propagation velocity is extremely slow, which allows the time-varying signal to be fully presented at the given moment. The surface of the substrate can access the signal.

3) SAW devices can be beneficial made with fine processing technology. Therefore, the SAW device has a very good repeatability and consistency, easy to mass production [2]-[9]. And when using certain crystalline materials or composites, the SAW device has extremely high temperature stability.

In many cases, the sensing mechanism of surface acoustic wave is often due to the change of the load mass caused by the surface adsorption, which changes the velocity and frequency of the surface acoustic wave, and the surface acoustic wave mass transmission. The sensitivity $S_{m}$ of the sensor are usually defined as [10]:

$$
S_{m}=\frac{1}{f_{0}} \lim _{\Delta m \rightarrow \infty} \frac{\Delta f}{\Delta m}=\frac{1 \Delta f}{\rho h f_{0}}
$$

And $f_{0}$ is the initial frequency of the sensor, $\Delta m$ is change in quality load, $\Delta f$ is changes in frequency caused by changes in quality, $\rho$ is density of mass load, $h$ is the thickness of the mass load layer.

This paper gives a brief introduction of the published results in the world, according to the relevant papers at home and abroad.

\section{Surface Acoustic Wave Sensor Structure and Application}

\subsection{Rayleigh Wave Sensor}

Rayleigh wave sensors are also commonly referred to as surface acoustic wave 
sensing device. General use $128^{\circ} \mathrm{YX} \mathrm{LiNbO}_{3}$ or stone (X Spread) quartz chips and so on. The former has a high voltage coefficient, the latter temperature stability is better. Manufacture Rayleigh wave sensor process is relatively simple, so the most easily to achieve commercialization [11] [12] [13] [14] [15]. The Due to Rui, the particle shift of the wave is elliptical orbit displacement is normal component. At the time of liquid-solid interface propagation, it energy will leak to the liquid, not suitable for sensor detection of liquid media, so it can only be used for gas atmospheres. As the frequency of the sensor is significantly more effect by physical quantity, such as the determination of pressure will be affected by temperature impact. So often need to keep in constant temperature conditions for sensing, testing, or pressure and temperature at the same time [16] [17] [18] [19].

At present, part of the Rayleigh wave sensor has been relatively mature or has reached commercial requirements, such as has been used for drugs, gas, and dangerous or harmful gas detection and analysis; but in the chemical, biological or special gas sensor. Because of the need for different chemical gases, to have different selective sensitive film, still need to continue to improve and improve the detection sensitivity [19].

We are using a $128 \mathrm{YX} \mathrm{LiNbO}_{3}$ filament substrate with an $80^{\circ}$ frequency of about $100 \mathrm{M} \mathrm{Hz}$ aluminum electrode interdigital transducer $\left(\mathrm{WO}_{3}\right)$ thin film as the sensitive layer and ultra-thin gold (or platinum) as the catalytic film, the development of hydrogen sensor, the synthesis of hydrogen in the air concentration of $1 \%$ to detect, to obtain room temperature, the sensitivity of the lower detection is similar to that of the existing sensor when the temperature rises to $270^{\circ} \mathrm{C}$, the sensor frequency changes by about $100 \mathrm{kHz}$ [20].

\subsection{Tangential Horizontal Plate Mode Sensor}

Because of the development of environmental science and bioengineering and technology, the demand of detecting environmental pollution and biological material is increasing. The sensor is mainly used in the liquid environment, so the Rayleigh wave sensor was inappropriate; usually choose the tangential horizontal displacement of the acoustic mode. The pattern of the horizontal tangential wave pattern (SH-APM) and lamb waves, etc., is usually chosen for the tangential horizontal displacement.

Horizontal tangential plate wave sensor is a kind of acoustic waveguide, which limits the acoustic energy. The sensitivity of the acoustic energy in the sheet depends on the thickness of the plate. The sensitivity of the board is greater, so the higher the sensitivity of the perturbation. Its unique advantage is the upper and lower surfaces are tangential horizontal displacement, so the upper and lower surfaces can be used for sensing. Figure 1 shows the shear level plate mode sensor structure.

\subsection{Love Wave Sensor}

The Love wave sensor is in the horizontal shear surface wave device surface 


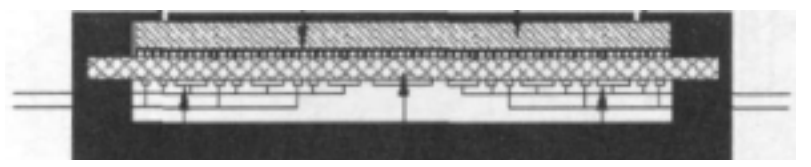

Figure 1. Shear level sound plate mode sensor structure.

Table 1. Comparison of sensitivity of several surface acoustic wave sensors.

\begin{tabular}{ccccccc}
\hline Device & Substrate & Wavelength Frequency & $\begin{array}{c}\text { Thickness of } \\
\text { waveguide/nm }\end{array}$ & $\begin{array}{c}\text { DMMP } \\
\text { concentration/ppm }\end{array}$ & $\begin{array}{c}\text { Frequency } \\
\text { shift } \Delta f \\
(H z)\end{array}$ \\
\hline $\begin{array}{c}\text { Love } \\
\text { wave 1 }\end{array}$ & AT-cut quartz & $40 \mathrm{~nm}$ & $115 \mathrm{MHz}$ & 15 & 0.35 & 1550 \\
$\begin{array}{c}\text { Love } \\
\text { wave 2 }\end{array}$ & AT-cut quartz & $40 \mathrm{~nm}$ & $111 \mathrm{MHz}$ & 15 & 0.35 & 2000 \\
SAW & ST-cut quartz & $32 \mathrm{~nm}$ & $98 \mathrm{MHz}$ & 30 & 0.3 & 180 \\
SH-APM & BT-cut quartz & $32 \mathrm{~nm}$ & $107 \mathrm{MHz}$ & 20 & 0.36 & 80 \\
\hline
\end{tabular}

covered with thin waveguide and ultra-thin metal catalyst film. The sound wave energy is concentrated in the thin waveguide, so the Love wave sensor can very sensitive to reflect the disturbance and change of the environment. Compared with the horizontal tangential plate mode [21], if the two achieve the same sensitivity. The tangential horizontal sound plate device is very thin, the mechanical strength is poor. And love wave device has a thick substrate, so strength of Love wave sensors is much better, but the process is more complex. As showed in the Table 1, Love wave sensors and SAW sensors, SH-APM sensors contrast the results. Love wave sensor is the surface acoustic wave sensor with the highest sensitivity, large mechanical strength of the sensor.

\subsection{Lamb Wave Sensor}

The Lamb wave sensor is propagated on the plate and can be seen as consisting of two rows of Rayleigh waves propagating on the upper and lower surfaces of the plate.

The illustration of Lamb wave propagation on the working surface of substrate showed in Figure 2.

$$
\theta_{R}=\sin ^{-1}\left(\frac{V_{W}}{V_{R}}\right)
$$

In the Equation (1), $V_{W}, V_{R}$ present the sound velocity in the liquid and phase.

If the thickness of the plate is greater than 2 times the wavelength, the two rows of Rayleigh waves are free to propagate. If the thickness of the plate is less than 2 times the wavelength, there are two groups of Lamb waves that can propagate independently of each other in the plate: symmetrical lamb waves and antisymmetric lamb waves. Usually there are infinite symmetric and antisymmetric Lamb waves propagating on the plate, but only the lowest order (zero order) of 


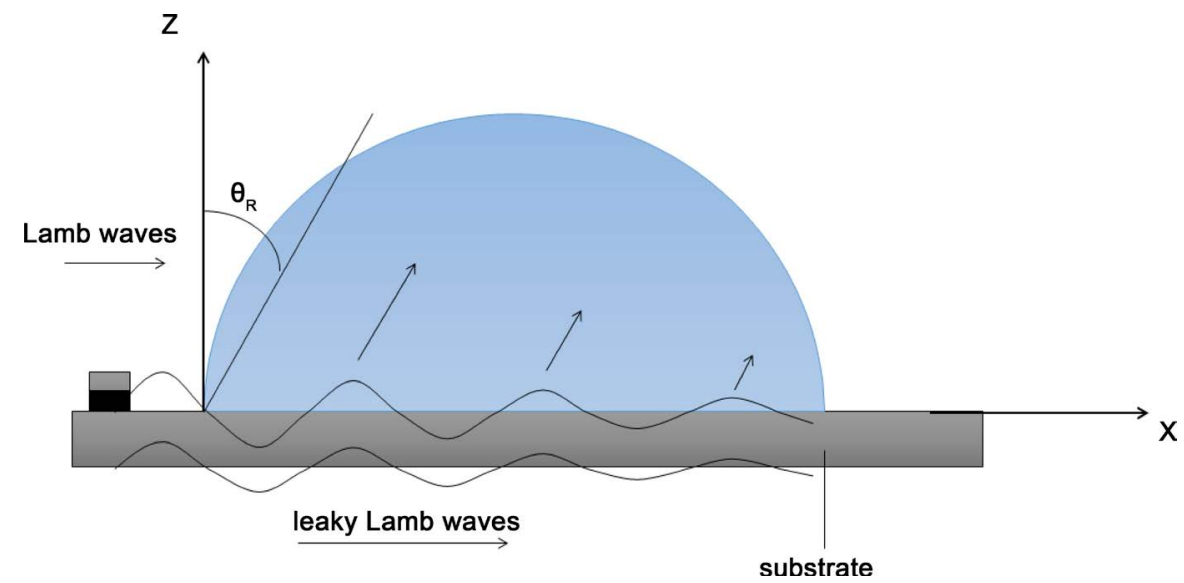

Figure 2. Schematic diagram of mode conversion of Lamb waves into compressional sound waves at the liquid-solid boundary of the droplet resulting in leaky Lamb waves.

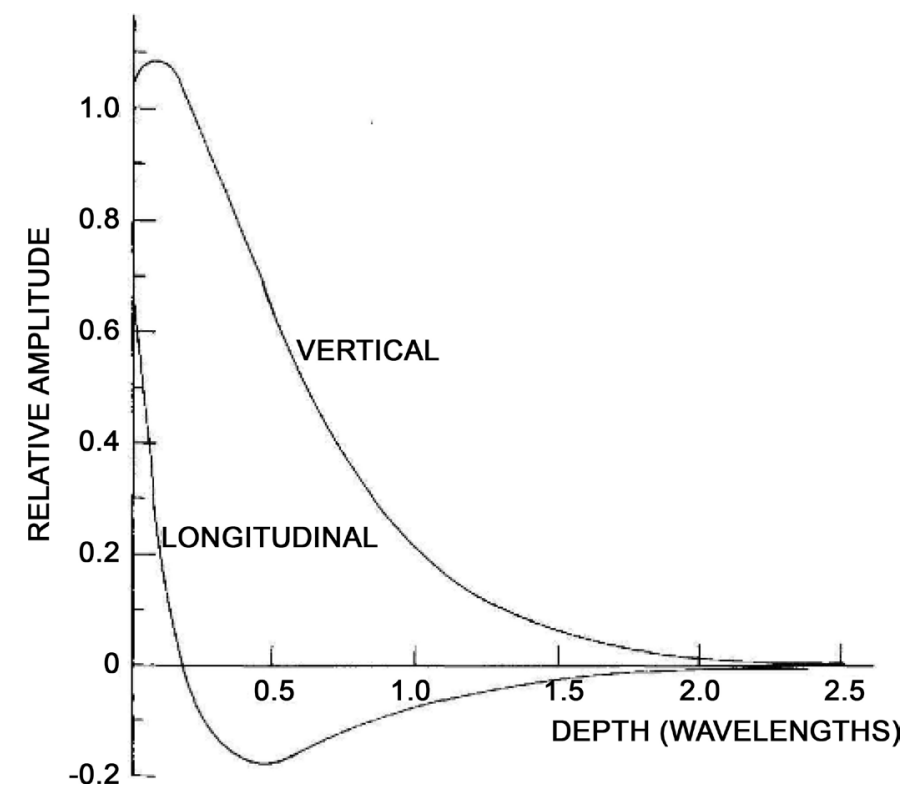

Figure 3. Displacement components as function of depth for an isotropic material.

the antisymmetric Lamb waves $\left(A_{0}\right.$ module) decreases monotonically with decreasing plate thickness. And $A_{0}$ module only has horizontal displacement, there is no vertical displacement, as long as the longitudinal wave velocity of the fluid medium is greater than $A_{0}$ module speed, can be used for fluid media sensing. Figure 3 shows the displacement components as function of depth for an isotropic material.

\section{Conclusion and Discussion}

SAW sensor is widely used in the industry because of its high sensitivity, high resolution, high stability, small volume and easy processing of output signals. SAW sensors with various structures and various materials have been developed by using various wave types. In the gas and liquid environment, SAW sensors were applied to the physical, chemical and biological aspects of the parameter 
sensing and detection. In the physical field, the detection object includes the quality, temperature, dew point, humidity, density, concentration, pressure, stress, torque, ultraviolet, voltage, etc. In chemical field, the detection object mainly includes a variety of chemical vapor, complexes, environment, the chemical composition of food or toxic and hazardous substances such as detection; In biology field, the detection object mainly includes cells, antibodies, bacteria, pathogens and other tests. Surface acoustic wave sensor detection of a variety of physical, chemical and biological field parameters are very accurate. With the development of high-tech, biological, chemical and medical, surface acoustic wave sensors will play an irreplaceable role in the future.

\section{References}

[1] Lin, S.-C.S., Mao, X. and Huang, T.J. (2012) Surface Acoustic Wave (SAW) Acoustophoresis: Now and Beyond. Lab Chip, 12, 2766-2770. https://doi.org/10.1039/c2lc90076a

[2] Miller, D.L., Smith, N.B., Bailey, M.R., Czarnota, G.J., Hynynen, K. and Makin, I.R.S.J. (2012) Overview of Therapeutic Ultrasonic and Safety Considerations. J UItrasound Med, 31, 623-634.

[3] Wiklund, M. (2012) Acousticfluidics 12: Biocompatibility and Cell Viability in Microfluidic Acoustic Resonator. Lab Chip, 12, 2018-2028. https://doi.org/10.1039/c2lc40201g

[4] Friend, J. and Yeo, L.Y. (2011) Microscale Acousticfluidics: Microfluidics Driven via Acoustic and Ultrasonics. Reviews of Modern Physics, 83, 647-704. https://doi.org/10.1103/RevModPhys.83.647

[5] Yeo, L.Y. and Friend, J.R. (2009) Ultrafast Microfluidics Using Surface Acoustic Waves. Biomicrofluidic, 3, 012002. https://doi.org/10.1063/1.3056040

[6] Wixforth, A.J. (2006) Acoustic Driven Programmable Microfluidics for Biological and Chemical Applications. Sensors and Actuators B: Chemical, 11, 399-405.

[7] Beyssen, D., Le Brizoual, L., Elmazria, O. and Alnot, P.S. (2006) Microfluidic Device Based on Surface Acoustic Wave. Sensors and Actuators B: Chemical, 118, 380-385.

[8] Zhang, G. and Li, Y. (2009) Regulating Charge and Excition Distribution in High-Performance Hybrid White Origanic Light-Emitting Diodes with n-Type Interlayer Switch. Nano-Micro Lett., 9, 37.

[9] Wang, Z. and Zhe, J. (2011) Paricle Intertial Focusing and Its Mechanism in Sperpentine Microchannel. Lab Chip, 11, 1280-1285. https://doi.org/10.1039/c0lc00527d

[10] Ogilvy, J.A. (1997) Precdicting Mass Loading Sensitivity for Acoustic Wave Sensors Operating in air. Sensors and Actuators, B42, 109. https://doi.org/10.1016/S0925-4005(97)00192-5

[11] Gedge, M. and Hill, M. (2012) Acoustic 17: Theory and Application of Surface Acoustic Wave Devices for Particle Manipulation. Lab Chip, 12, 2998-3007. https://doi.org/10.1039/c2lc40565b

[12] Fu, Y.Q., Luo, J.K., Du, X.Y., Flewitt, A.J., Li, Y., Markx, G.H., Walton, A.J. and Milne, W.I. (2010) ZnO Thin Films Prepared at Low Annealing Termperatures, from a Novel, Simple Sol-Gel Precursor Solution. Sensors and Actuators B: Chemical, 143, 606-619. https://doi.org/10.1016/j.snb.2009.10.010

[13] Tan, M.K., Friend, J.R. and Yeo, L.Y. (2007) Direct Visualization of Surface Acous- 
tic Waves along Substrates Using Smoke Paeticles. Applied Physics Letters, 91, 224101. https://doi.org/10.1063/1.2814054

[14] Ding, X., Shi, J., Lin, S.-C.S., Yazdi, S., Kiraly, B. and Huang, T.J. (2012) Tunable Patterning of Microparticles and Cells Using Standing Surface Acoustic Waves. Lab Chip, 12, 2491-2497. https://doi.org/10.1039/c2lc21021e

[15] Hashimoto, K. and Yamaguchi, M. (2001) SAW Excitation Propagation under Periodic Metallic Grating Structures. IEEE Transactions on Ultrasonics, Ferroelectrics, and Frequency Control, 48, 1181-1188. https://doi.org/10.1109/58.949731

[16] Schröder, C.T. and Scott Jr., W.R.J. (2001) On the Complex Conjugate Roots of the Rayleigh Equation: The Leaky Surface Wave. The Journal of the Acoustical Society of America, 110, 2867-2877. https://doi.org/10.1121/1.1419085

[17] Adler, E. (1994) SAW and Pseudo-SAW Properties Using Matrix Methods. IEEE Transactions on Ultrasonics, Ferroelectrics, and Frequency Control, 41, 876-882. https://doi.org/10.1109/58.330269

[18] Auld, B. (1973) Auld Acoustic Fields and Waves in Soild. Acoustic Fields and Waves in Solids. Wiley Press, New York.

[19] Köster, D. (2007) Evaluation of the Successive Approximations Methods for Acoustic Streaming Numerical Simulations. SIAM Journal on Scientific Computing, 29, 2352-2380. https://doi.org/10.1137/060676623

[20] Ippolito, S.J., Kandasamy, S., Kalantar-Zadeh, K., et al. (2005) Highly Sensitive Layered $\mathrm{ZnO} / \mathrm{LiNbO}_{3} \mathrm{SAW}$ Device with in OX selective layer for $\mathrm{NO}_{2}$ and $\mathrm{H}_{2}$ Gas Sensing. Sensors and Actuators, B108, 154.

https://doi.org/10.1016/j.snb.2004.11.092

[21] Wiklund, M., Green, R. and Ohlin, M. (2012) Acoustofluidics 14: Application of Acoustic Straming in Microfluidic Devices. Lab Chip, 12, 2438-2451.

https://doi.org/10.1039/c2lc40203c

Open Access Library

\section{Submit or recommend next manuscript to OALib Journal and we will pro-} vide best service for you:

- Publication frequency: Monthly

- 9 subject areas of science, technology and medicine

- Fair and rigorous peer-review system

- Fast publication process

- Article promotion in various social networking sites (LinkedIn, Facebook, Twitter, etc.)

- Maximum dissemination of your research work

Submit Your Paper Online: Click Here to Submit

Or Contact service@oalib.com 